\title{
LA PERCEPCIÓN DE LOS ESLABONES DE LA CADENA DE VALOR Y SUMINISTRO DE LA EDUCACIÓN SUPERIOR: VISTA DESDE LOS ACTORES VINCULADOS
}

Margarita Elizabeth Díaz, Universidad Nacional Autónoma de Honduras, Instituto de Investigaciones Económicas y Sociales (IIES UNAH), Ciudad universitaria edificio C2 primer piso. Tel./Fax: 504-22391849

Correo electrónico: mdiaz@iies-unah.org

\section{RESUMEN}

Se realizó una consulta a las instituciones vinculadas a la educación superior y la demanda de profesionales universitarios: i.- Universidades públicas y privadas, ii.- Instituciones gubernamentales central y descentralizadas, iii.- Instituciones privadas gremiales o productivas, todas ellas vinculadas a los eslabones que conforman "La cadena de valor y suministro de la educación superior".

Los eslabones de la cadena de valor identificados fueron: 1.- Proveedores de insumos: Graduados de secundaria, familia, gobierno y organizaciones privadas, escuelas del profesorado, colegios; 2: Educación Superior: Universidades, centros de investigación; 3: Demandante Intermedio: profesionales, cooperantes y resultados de investigación; 4: Demandante Final: Sociedad y empleadores. Todos estos eslabones vinculados en una dinámica funcional de relaciones de oferta y demanda de profesionales universitarios desde su formación hasta su ubicación dentro del mercado laboral.

Se buscó determinar las vinculaciones existentes entre las instituciones a las que ellas representaban y el resto de las instituciones dentro de la cadena, de manera de ver las interacciones y de cómo generan insumos al siguiente eslabón de la misma. Lo cual se tornó aún más interesante porque dependiendo de posición del eslabón dentro de la cadena, si ofrece insumos que lo que demanda, o si por otro lado, ejerce más demanda de los profesionales que salen de las universidades, absolutamente todas las instituciones concluyeron interesantes observaciones de cómo están las universidades del país actualmente y en qué aspectos se debería mejorar ofrecer mejores profesionales universitarios.

Palabras claves: Cadena de valor, eslabones, educación superior, mercado laboral. 


\title{
PERCEPTION OF THE LINKS OF THE CHAIN OF VALUE AND SUPPLY OF THE HIGHER EDUCATION: VIEW FROM THE LINKED ACTORS
}

Margarita Elizabeth Díaz, Universidad Nacional Autónoma de Honduras, Instituto de Investigaciones Económicas y Sociales (IIES UNAH), Ciudad universitaria edificio C2 primer piso. Tel./Fax: 504-22391849

Correo electrónico: mdiaz@iies-unah.org

\begin{abstract}
A consultation was made to the institutions involved in higher education and demand of university professionals: i.- Public and private universities, ii.- Central and decentralized governmental institutions, iii.- Gremial private institutions or all of those involved in the links that form "The value chain supply of higher education."
\end{abstract}

The links of the value chain are: i.- Provision suppliers: Those graduated from high school, families,

E\&A the government and private organizations, teaching education schools, high schools; 2.- Higher Education: Universities, research centers; 3.- Intermediate petitioner: Professionals, aid workers, research results; 4.- Final petitioner: Society, employers. All the links are in a functional dynamics of relationships of offer and demand of university professionals from their formation up to their positioning within the labor market.

It was necessary to determine the existing connections between the institutions to which they represented and the remaining institutions within the chain, in order to see the interactions and the way provisions are generated for the following link. Which became even more interesting because depending on the position of the link within the chain, if it offers more provisions than demand, or if, on the other hand, it exerts more demand of university professionals, absolutely all the institutions concluded interesting observations on how the country's universities are actually doing and on which aspects should be improved to offer better university professionals.

Palabras claves: Value chain, links, higher education, labor market. 


\section{OBJETIVO DEL ANÁLISIS}

Consultar a las instituciones de la triple Hélix: Universidades, Gobierno y empresa privada sobre la percepción de la educación superior y los profesionales universitarios, desde el eslabón al cual pertenecen, de manera de ver las vinculaciones, interacciones, oferta y demanda de estos profesionales en los diferentes sectores del mercado laboral.

\subsection{LA EDUCACIÓN SUPERIOR Y LA VINCULACIÓN CON EL MERCA- DO DE TRABAJO}

La vinculación del mercado laboral en la educación superior y viceversa, Mungaray (2001:3) menciona que:

El mercado laboral esta expuesta a una serie de presiones económicamente mas globales: i.- Los cambios bruscos que se dan en las necesidades económicas, sociales y políticas modifican las circunstancias y situaciones de estos mercados laborales, ii.- La modernización de las empresas y las instituciones gubernamentales sin equilibrios locales, y la descentralización gubernamental de funciones centrales sin soltar la toma de decisiones, iii.- Un nuevo tipo de individualismo y promoción de una acción política más participativa, con más actores en los campos de los bienes públicos, el capital humano y de la educación superior.

Según Teichler, (1999) la empleabilidad se puso en la agenda política con las siguientes tendencias: i.De empleo regular hacia una forma de trabajo más flexible y precaria, ii.- De un paradigma elitista y de escasez hacia un paradigma de masa y abundancia, iii.- De una sociedad de formación 'pre-carera profesional' hacia una sociedad de aprendizaje de toda la vida, iii.- De un mercado de formación y trabajo nacional hacia un escenario global o internacional. En este ir y venir de circunstancias mas bien globales la empleabilidad de los graduados universitarios se vuelve en general mas interesante sobre el objetivo de la educación superior con relación al mercado laboral. (P.185)

La vinculación entre el mercado de trabajo y los profesionales de las diferentes disciplinas del conocimiento pasan por la intermediación de las instituciones de educación superior (IES). Las universidades captan las necesidades de los sectores demandantes de la sociedad y lo que estas 
ofrecen del nivel profesional. "El mercado laboral como una relación social es la que permite y regula los intercambios entre los agentes económicos y sociales" Mungaray, (2001), esto incluye las vinculaciones de diferentes instituciones o figuras sociales que se relacionan en el proceso de formación de profesionales.

Estas figuras sociales intervienen en un continuo en todo el proceso de educación terciaria, lo que asegura no solo la permanencia de una educación formal, sino de acuerdo a las tendencias actuales, a los requerimientos que exigen las instituciones privadas, productivas y del sector público; de manera que el proceso de educación universitaria aunque se da por si misma, no esta aislada de otras instancias e instituciones que intervienen ya sea como proveedoras o como demandante de los profesionales que son producto de las instituciones.

\subsection{CADENA DE VALOR DE LA EDUCACIÓN SUPERIOR}

De acuerdo al enfoque de Porter. Michael y en una contextualización mas amplia de una cadena de valor se puede decir que "la cadena de valor tiene una perspectiva más interna, centrándose en la institución misma, incluye una orientación horizontal de las relaciones interde-sectores, que agrupa en la categoría que denomina las actividades de apoyo; y que enfatiza las interrelaciones de la institución con otras instituciones que son sus proveedores y quienes son sus clientes, a través de los "eslabones" que denomina "logística de entrada" y "logística de salida".

Desde esta perspectiva la cadena de valor en el contexto de la educación superior vinculada al mercado laboral, se puede decir que esta es una serie de procesos desde: i.- Las instituciones vinculadas al mismo, ii.- El proceso de formación de las universidades, iii.- Los sectores productivos que demandan profesionales universitarios; en dicho proceso cada uno de las instituciones y actores vinculados agregan valor dentro de la cadena.

El sistema de valor forma parte de una serie de actividades en el cual participan un grupo de actores ubicados en diferentes posiciones, en el que al menos tres cadenas de valor se identifican: i.- Cadena de valor de los que proveen: Instituciones que aportan los insumos esenciales a la propia cadena, estos 
suministros influyen en las universidades y/o en sus capacidades de diferenciación, ii.- Cadena de valor de los canales: Las universidades que entregan profesionales formados y graduados a los demandantes de estos, todo lo que impactará a nivel de valor agregado en el mercado laboral, iii.Cadena de valor de los demandantes: Los diferentes sectores de la sociedad, en la cual se da la diferenciación por excelencia, porque es en función de la calidad de los profesionales que determina las necesidades de la sociedad que los solicita.

En el contexto de este artículo los actores que participan de la cadena de valor se llamaran eslabones, en la Figura 1 se puede observar los diferentes eslabones dentro de la cadena y

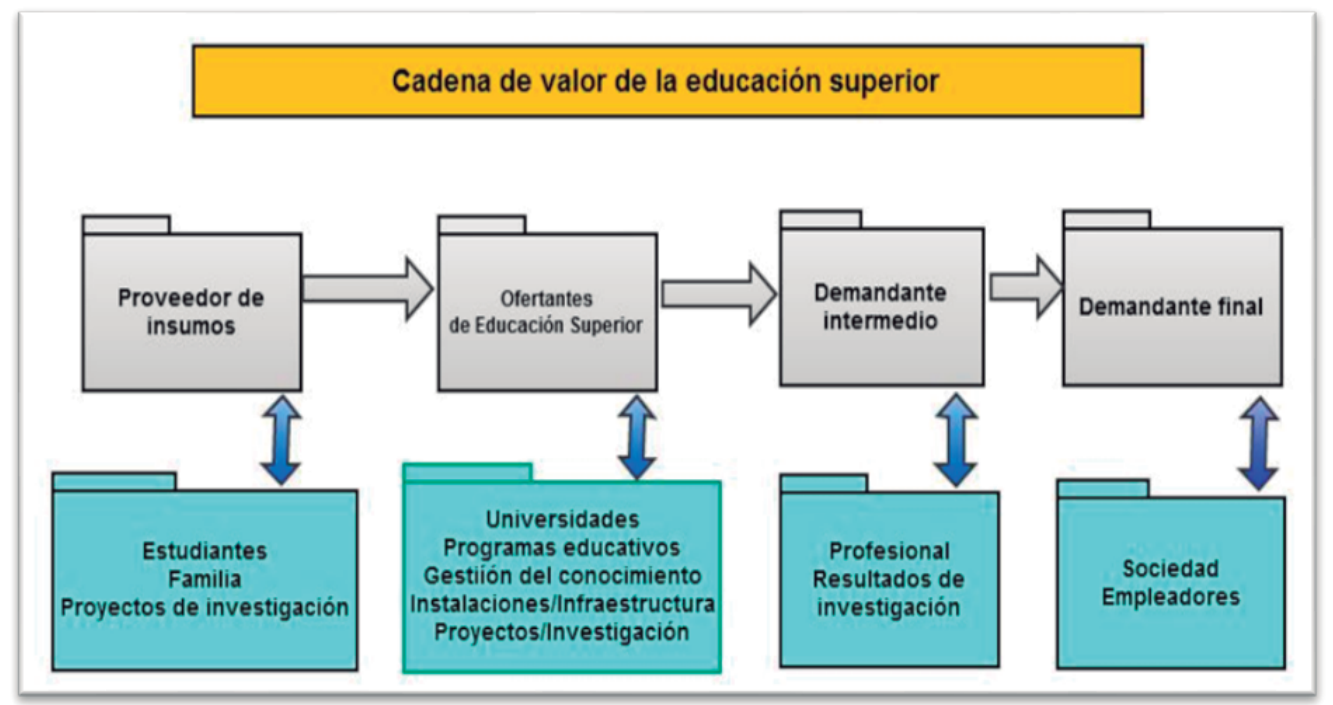

Figura 1. Cadena de valor de la educación superior. De Demanda y Oferta de Educación Superior: Integración Total de la Cadena de Valor y las Cadenas de Suministro [Manuscrito no publicado] (p. 13), por C. Ortega, 2011.

En la cadena de valor describe claramente que eslabones participan del proceso y que instituciones la alimentan, desde los proveedores de insumos, quienes dan al eslabón de educación superior los estudiantes que se formaran profesionalmente y serán devueltos a la sociedad que los demanda, para ubicarlos en el mercado laboral en todas las áreas que el país necesita. 


\section{METODOLOGÍA}

\subsection{ESTRATEGIA METODOLÓGICA}

Se estableció una estrategia metodológica de evaluación centrada en el desarrollo de capacidades de las instituciones para identificar la articulación oferta y demanda de profesionales universitarios con el fin de conocer el sistema de educación superior en el país. En este sentido, se planteó dos propósitos: i.- Definir conceptos y estimular una reflexión más amplia sobre la oferta de profesionales universitarios y la demanda desde el sector productivo empresarial de estos, basada en la articulación universidad-empresa, y ii.- Fortalecer la generación de información y ver los cambios que se generan en el sistema educativo superior. Estos propósitos se basan en los niveles de participación.

Se utilizó la técnica de los grupos focales basados en la conformación del sistema de valor y suministro de la educación superior: 1: Proveedores de Insumos, 2: Educación Superior (Ofertantes), 3: Demandante Intermedio; Intermedio y 4: Demandante Final, en dos fases de grupos focales: Primera fase: Grupos focales exploratorios, en los cuales se exploró aspectos generales de cada uno de los eslabones dentro del sistema de educación superior. Segunda fase: grupos focales de duelo e integrador en los cuales se confrontaron los grupos para identificar la relación entre los diferentes eslabones y los puntos de encuentro entre ellos.

\subsection{POBLACIÓN, SECTORES Y PARTICIPANTES}

La población de los grupos focales fueron las universidades, las empresas, gremios profesionales y otras instituciones que están vinculadas a la oferta educativa terciaria y a la demanda de profesionales universitarios. Esta población se resume en los siguientes sectores: i.- Sector educativo: Los establecimientos de educación superior nacionales como privados de Tegucigalpa; ii.- Sector público y privado: Las instituciones del Gobierno Central, otras instituciones ligadas a la contratación de profesionales universitarios como ser gremios profesionales y empresas; y iii.- Sector productivo-empresarial: Los gremios organizados y vinculados muy directamente con el quehacer productivo del país, como grandes empresas y pequeñas. 


\section{INSTITUCIONES PARTICIPANTES}

\section{Para los grupos focales exploratorios}

Las instituciones que participaron fueron las siguientes: i.- Proveedores de insumos (cooperantes, educación media, familia):-Secretaría de Educación, Secretaría de Finanzas: Secretaría de Finanzas: SEFIN, Dirección General de Presupuesto: DGP, e Instituto Nacional de Formación Profesional: INFOP.

ii.- Los ofertantes (Educación superior): Universidad Nacional Autónoma de Honduras: UNAH, Dirección de Educación Superior: DES, la Vice-Rectoría Académica: VRA y Centro Universitario Regional Litoral Atlántico: CURLA; Universidad Pedagógica Nacional Francisco Morazán: UPNFM, Unidad Externa de Medición de la Calidad de la Educación: UMCE; Universidad Tecnológica Centroamericana: UNITEC, Centro Universitario Tecnológico: CEUTEC; Universidad Tecnológica de Honduras: UTH; Universidad Metropolitana de Honduras: UMH; y Escuela Agrícola el Zamorano: EAZ.

iii.- Demandantes intermedios (cooperantes, familia, propiedad intelectual): Organismo de Estados Americanos: OEA, Red Carolina de Honduras, Asociación Educación y Familia: AEF, Asociación de estudiantes universitarios, Asociación de Estudiantes de la UNAH, Becarios y Prestatarios: ASEBEP, Profesionales, Dirección General de Propiedad Intelectual: DIGEPI.

iv.- Demandante final (ES, empleador y gobierno): Secretaria de Planificación: SEPLAN; Secretaria de Trabajo y Seguridad Social: STSS; Observatorio Mercado laboral: OML; Secretaría de Agricultura: SAG, Dirección de Ciencia y Tecnología Agropecuaria: DICTA, Banco Central de Honduras: BCH, Federación Nacional de Agricultores y Ganaderos de Honduras: FENAGH, Comisión Nacional de bancos y Seguros: CNBS.

Para los grupos focales de duelo e integrador

Proveedores de insumos (cooperantes, educación media, familia): Secretaría de Educación 
Secretaría de Finanzas: SEFIN, Secretaria de Planificación: SEPLAN, HONDURAS GLOBAL INFOP, Instituto Nacional de Formación Profesional.

Ofertantes (Educación superior): Universidad Tecnológica Centroamericana: UNITEC, Universidad Metropolitana de Honduras: UMH, Universidad Tecnológica de Honduras: UTH, Universidad Pedagógica Nacional Francisco Morazán: UPNFM, Universidad Nacional Autónoma de Honduras UNAH: Vice-Rectoría Académica: VR, Dirección de Educación Superior: DES.

Demandantes intermedios (cooperantes, familia, propiedad intelectual): Representante de Cooperantes (Fullbright), Secretaría de Planificación: SEPLAN, Representante Enlace Honduras Global, Asociación de Estudiantes, Becarios y Prestatarios de la UNAH: ASEBEP, Representante profesional de post-grados.

Demandante final (ES, empleador y gobierno): Secretaría de Agricultura: SAG, Comisión Nacional de Banca y Seguros: CNBS, Dirección de Propiedad Intelectual: DPI, Universidad Nacional autónoma de Honduras: UNAH, Vice-Rectoría de Asuntos Académicos: VRA.

\section{Temas y preguntas de los grupos focales}

La temática desarrollada en los grupos focales estaba concentrada en los siguientes aspectos: i.- La articulación de las instituciones de educación superior y sus suministros, ii.- Los procesos internos de investigación, desarrollo e innovación ( $\mathrm{I}+\mathrm{D}+\mathrm{i})$, iii.- La vinculación sociedad (demanda de ES) Educación Superior, iv.- Las relaciones entre los diferentes eslabones de la cadena de valor, v.- Las características y la naturaleza de la oferta y demanda de profesionales de educación superior, vi.- Las relaciones de la educación superior con el nivel educativo medio, y vii.-la dinámica al interior del sistema educativo terciario.

Las preguntas que se abordaron están incluidas en la sección de resultados 


\section{Resultado de los grupos focales \\ Grupos focales exploratorios}

\section{1.- ¿Quiénes anteriormente a este evento tenían conocimiento del sistema integrado de valor y suministro de la educación superior?}

Todos los eslabones tenían conocimiento del concepto, sin embargo, o lo conocían en otros campos de aplicación o solo como cadena de valor, no de suministro, en general se puede concluir que los participantes si conocían el modelo de valor y suministro de manera general, el eslabón de educación superior mencionó que debe de estandarizarse porque de ahí salen los planes de estudios de las diferentes carreras.

También este eslabón de educación superior opinó que en la práctica el modelo se ha visto de manera aislada, pero debe integrarse y sistematizarse viendo cada una de sus fases, lo cual lo ubica en contexto inmediatamente del modelo.

En este contexto, los diferentes participantes se ubicaron en el eslabón al cual pertenecían dentro del modelo en el contexto de la educación superior y sus vinculaciones con el resto de los eslabones que participan desde el inicio hasta el final de la cadena de valor y suministro de la misma.

\section{2.- Desde la entidad a la cual Ud. pertenece ¿considera pertinente el planteamiento de este modelo para el estudio de la ES?}

Todos sin excepción opinaron que el modelo propuesto es más que pertinente por varias razones: i.Los proveedores de insumos opinaron que el sistema educativo merece atención y dentro de él los roles que cada uno de los eslabones que tiene en el sistema; ii.- La educación superior aseguró que se convierte en una necesidad y para tener una visión de país, iii.- Los demandantes Intermedios dijeron que se puede impulsar el desarrollo a través de la educación terciaria y iv.- Los demandantes finales aseguraron que el modelo es aplicable a toda actividad productividad y educativa. 
Relacionado con estas respuestas, existen otras razones que sostienen la pertinencia del modelo de valor y suministro de educación superior: i.- Cualquier modelo se puede implementar, porque se puede desarrollar según los proveedores de insumos; ii.- Los planes de estudios son obsoletos, deben entonces actualizarse opinaron los de la educación superior; iii.- Este modelo genera la sinergia que pretende el sistema de la cuádruple hélice sostuvieron los demandantes intermedios; se avanza con los nuevos procesos de globalización afirmaron los demandantes finales de la cadena de valor y suministro de la ES.

\section{3.- ¿Cuáles son las fortalezas del modelo?}

El eslabón de la educación superior aseguró que el modelo genera una institucionalidad y permite integrar o complementar para tomar mejores decisiones; los demandantes intermedios opinaron que el modelo puede identificar y vincular cada eslabón; los demandantes finales aseguraron que hay un vínculo entre valor y suministro.

El modelo permite: i.- Identificar cuellos de botella que hay en el sistema de educación superior, ii.- El tipo de profesionales que necesita el país, iii.- Ver desde la óptica de la demanda nacional, regional y globalmente los profesionales que se necesitan, iv.- Puede dar una mirada general de lo que pasa en el sector público y privado y v.- Establecer alianzas estratégicas entre diferentes instituciones.

\section{4.- ¿Qué limitaciones observa en el modelo?}

El eslabón de los proveedores de insumos concluyeron que la falta de vinculación de los eslabones no permite una mejor divulgación de profesionales en el mercado laboral; la educación superior mencionó aspectos importantes como que el modelo no considera el nivel pre-universitario, no vincula todas los eslabones de manera de orientar al estudiante a profesionalizarse en las áreas que el país necesita, que en gran parte depende de la demanda del mercado laboral, sobre todo de las grandes empresas.

Por otro, los demandantes Intermedios mencionaron algunos aspectos importantes como los recursos 
disponibles para implementar el modelo y que la investigación sea visible en este contexto; los demandantes finales opinaron que no todos los factores están incluidos en el modelo, y que algunos factores que definitivamente están fuera del modelo son: i.- El pensum académico, ii.- La acreditación regional, iii.- La demanda del mercado laboral, iv.- La vinculación universidad y gobierno y v.- Las fugas de profesionales formados en el país.

\section{5.- ¿Cuáles son los factores que se encuentran al margen del modelo?}

El eslabón de proveedores de insumos mencionaron sobre la inducción vocacional a los estudiantes para la elección de carrera universitaria y ver a la educación superior como un ente que ayuda a resolver a los problemas nacionales; el eslabón de la educación superior consideró que los factores que están al margen del modelo son: i.- Las políticas institucionales universitarias, ii.- La disponibilidad de recursos financieros y iii.- Otros recursos.

\section{6.- Considera ¿Qué el nivel de eficiencia se mantiene en cada uno de los eslabones?}

El eslabón de los proveedores de insumos opinaron que hay una excelente comunicación y participación directa en el nivel de eficiencia de cada uno de los eslabones de la cadena de valor, lo que da lugar a que la universidad tome un papel protagónico en la solución de problemas por medio de la innovación; la educación superior consideró que los profesionales están saliendo o bien formados o mal formados dependiendo de cómo vienen preparados los estudiantes de la secundaria.

Los demandantes intermedios consideraron que falta información y comunicación para los estudiantes, que hay que promocionar el modelo aún más; los demandantes finales manifiestan que el modelo es ambicioso, que se debe priorizar el desarrollo del modelo para hacerlo sostenible en el tiempo, asimismo, como establecer una serie de indicadores para dar información a quienes lo consulten. 


\section{Grupos focales de duelo}

\section{Grupo Focal de Duelo No 1: 1: Proveedores de insumos y 2: Ofertantes (Educación Superior)}

De acuerdo a la compilación de respuestas dadas por las instituciones en el grupo focal de duelo entre los proveedores de insumos y los ofertantes, o se las instituciones de educación superior, las preguntas y respuestas (Díaz, 2012a) son las siguientes:

\section{Pregunta general}

\section{¿Cómo percibe la articulación entre la educación superior y el mercado} laboral?

La educación superior y mercado laboral esta desarticulado, adicionalmente a esta desarticulación el sistema educativo nacional en su conjunto esta desarticulado también, lo que genera un sistema educativo en general que no se vincula y con grandes falencias.

Por otro parte, en el mercado laboral existe una descompensación entre el salario percibido y el nivel educativo logrado, ya que el mercado laboral no valora esta jerarquía de formación académica.

\section{2: Ofertantes (Educación Superior) $\rightarrow$ 1: Proveedores de insumos}

\section{Como proveedores. ¿Consideran que están brindando los insumos necesarios a la ES para que los resultados sean de calidad? (Tanto estudiantes como proyectos)}

$\mathrm{Al}$ estudiante no solo se le ve como un insumo de la educación superior, sino como un beneficiario también de la misma. El estudiante graduado de educación media en todas sus modalidades no tiene las suficientes capacidades y competencias necesarias requeridas.

La calidad de la educación media es cuestionada y se enfatiza que se están transfiriendo los errores de la educación media a las universidades, lo que implica que los graduados de educación media no van tan preparados académicamente para enfrentar los retos de una educación superior. En los centros 
educativos de educación media no solo hay un problema de calidad, sino una cantidad exagerada de alumnos.

Por otro lado, INFOP como una institución de formación profesional, es vista como una institución que fortalece la educación media, al proveer formación a estudiantes de media en una gama de talleres y cursos que son mas bien capacitaciones técnicas en algunos oficios y que pueden generarle al estudiante de educación media un ingreso o un empleo.

\section{1: Proveedores de insumos $\rightarrow$ 2: Ofertantes (Educación Superior)}

\section{Pregunta general}

\section{¿Cómo percibe el rendimiento de la ES en función de los recursos utilizados y los resultados obtenidos? (Tanto profesionales como proyectos)}

La investigación no está orientada en los centros de educación superior, en especial en centros universitarios privados. Se requieren de una política universitaria homogénea con lo que respecta a la investigación en cuanto a líneas de investigación. Tampoco hay una tradición investigativa y a los profesionales que se les asigna tarea de investigación. Especialmente las universidades privadas no tienen los recursos para desarrollar la investigación.

No existen sistemas de monitoreo y evaluación del conocimiento impartido en las aulas de clase, ni tampoco del desempeño del docente, ni en las universidades públicas, ni las privadas.

El potencial de desarrollar competencias y habilidades en las universidades tiene limitaciones económicas, logísticas y potencialidad física que permita que los estudiantes expandir no solo sus actividades académicas, sino también su actividad de recreación y potencialidades artísticas. En este sentido, las universidades surgieron de manera desorganizada, sin tener toda la infraestructura ni los recursos adecuados que ofrecer a los estudiantes. Sin embargo, se están realizando esfuerzos para fortalecer la educación superior. 


\section{2: Ofertantes (Educación Superior) $\rightarrow$ 1: Proveedores de insumos}

\section{Creen que todas las universidades poseen potencial para poder desarrollar competencias y mejores habilidades en los servicios que brindan los proveedo- res de insumos}

En cuanto a la utilización de los recursos todas las universidades consultadas creen que están aprovechando bien sus recursos disponibles, ya sean estas: i- Infraestructura, ii.- Recursos tecnológicos, iii.- Mayor cobertura de estudiantes.

La UNAH por medio de la Cuarta Reforma Universitaria ha tratado de optimizar los recursos disponibles y el resultado logrado, se ha hecho un esfuerzo por hacer un balance de manera de obtener una eficiencia en los servicios que se prestan. Sin embargo, no hay una evaluación de que si efectivamente la utilización de esos recursos y la relación a los resultados.

\section{1: Proveedores de insumos $\rightarrow$ 2: Ofertantes (Educación Superior)}

\section{Considerando las cadenas de docencia e investigación, ¿Observa que las ins- tituciones de educación superior desarrollan adecuadamente las actividades fundamentales?}

En general piensan que si hacen la labor docente, ya que se exigen a los catedráticos que tengan los requisitos requeridos para cada nivel académico en el cual ellos impartan las clases.

La capacitación continua de los docentes se realiza con el objetivo de que ellos aprendan a ser maestros con las metodologías adecuadas al nivel, esto se realiza por medio de institutos de superación personal. Ser docente requiere tener no solo la vocación, sino las aptitudes que lo perfilen como tal. La certificación del docente en torno a los elementos pedagógicos se da a través de los métodos de certificación que existen.

La investigación pura y aplicada que realizan algunas universidades es muy poca, ya que se carece de suficientes estímulos que produce y los escasos resultados hace que no haya estímulo para investigar en algunas universidades. 


\section{Grupo Focal de Duelo No 2: \\ 2: Educación Superior y 3: Demandante Intermedio}

En base a las respuestas compiladas de este grupo focal desde las universidades y los demandantes (Díaz, 2012b) las respuestas son las que a continuación de describen:

\section{Pregunta general}

\section{¿Cómo percibe la articulación entre ES y el Demandante intermedio?}

En cuanto a la articulación educación superior y mercado laboral los centros educativos de educación superior consideran que no hay articulación por varias razones: i.- Por la falta de incentivos orientadores para los estudiantes, ii.- Los gremios profesionales no dan información a sus agremiados adonde ubicarse en el mercado laboral, iii.- -No hay seguimiento a los graduados, iv.- No se percibe desde el demandante intermedio a la educación superior, v.- La actual oferta académica no solventa las necesidades sociales.

En cuanto a la oferta educativo del nivel varios aspectos importantes salen a relucir: i.- Las universidades siguen ofreciendo las mismas carreras tradicionales ii.- No se atienden las necesidades del demandante intermedio, y las necesidades que el mercado laboral y la sociedad requieren, iii.- No se ofrecen carreras hacia las necesidades de las diferentes regiones del país, que requieren profesionales en ciertas áreas del conocimiento específicas, iv.- Hay dificultad en los procesos de organización de la actividad académica.

\section{2: Educación Superior $\rightarrow$ 3: Demandante Intermedio ¿Están siendo desarrollados correctamente los programas curriculares en ES?}

Los programas curriculares si están estructurados pero deben renovarse ya que estos no están diseñados de acuerdo a lo que el desarrollo de un país requiere, debe de someterse a un proceso de revisión para actualizarlos y adaptarlos a las verdaderas necesidades del país. Aunque hay que reconocer que se ha mejorado en los procesos de acreditación de universidades, evaluaciones a los maestros y la autoevaluación. 
Se debe de realizar revisiones curriculares de manera permanente, hay tres aspectos que inciden en dicha actualización orientados a: i.- Actualizaciones e innovaciones de la currícula, ii.- Aplicación de una metodología de la enseñanza teórica con la práctica, iii.- Elementos de emprendedurismo, que le permita al estudiante pasar de ser empleado a empleador, dadas las condiciones del empleo en el país.

Los procesos de politización e intereses de diversa índole en la UNAH y el resto de las universidades públicas y privadas inciden en el acondicionamiento de sus pensum académicos. La UNAH que rectora la educación superior es la responsable de regular las universidades del nivel para que adecuen y actualicen los pensum académicos.

\section{3: Demandante Intermedio $\rightarrow$ 2: Educación Superior}

\section{Qué tipo de dificultades se presentan en la acreditación de títulos, certificación de programas (pre-grado, post-grado) y registro de resultados de investiga- ción?}

Si se cuenta con una infraestructura básica y laboratorios, sin embargo, la población estudiantil va en continuo aumento y demanda sobre esta infraestructura ya instalada, lo que la hace insuficiente y puede volverse obsoleta, sino se hace un esfuerzo de modernizarlas y aumentarlas de acuerdo a la demanda de estudiantes.

En este contexto, no todas las universidades cuentan con laboratorios y salas especializadas de acuerdo a las áreas de conocimiento, no se puede concluir que en todos los centros universitarios presenciales o a distancia, universidades principales y centros universitarios regionales cuentan con la misma calidad de infraestructura.

Por otro lado, las tecnologías de información también varían de acuerdo a las universidades, hay que mejorar no solo la accesibilidad, sino los recursos informáticos que mejoren el dominio de los medios digitalizados y los intercambios intra y extra-curriculares, que solo con acceso a internet pueden lograrse, incluyendo la comunicación más rápida. Así mismo, como el acceso a grandes cantidades de información académica y bases de datos de las mejores universidades del mundo. 


\section{2: Educación Superior $\rightarrow$ 3: Demandante Intermedio}

\section{¿Considera que ES cuenta con las instalaciones e infraestructura óptima para desarrollar las capacidades de las carreras?}

Los procesos de certificación de programas de pre-grado y post-grado son ineficientes, burocráticos, deberían implementarse procesos más ágiles y rápidos que permitan a los graduados certificar mejor y más rápidamente sus títulos.

Los trámites para aprobar planes de estudio también son burocráticos, engorrosos, esto en cuanto a su aprobación; pero por otro lado, puede ser sometido a un cambio en su plan de estudio, el programa que empieza no es el mismo con el que se gradúa, dándose una serie de inconsistencias.

En otra línea, si el plan de estudios es pertinente, pero no lo asimila es docente y no lo pone en práctica, no podrá implementarse.

Por otro lado, el proceso de investigación no se percibe desde el demandante intermedio como que sea importante para las universidades, y sus resultados deberían de publicarse y tener accesibilidad de toda la sociedad en su conjunto.

\section{3: Demandante Intermedio $\rightarrow$ 2: Educación Superior}

\section{Se vinculan las universidades para mejorar la percepción del valor de lo que entregan en - docencia y en investigación}

Si existe una vinculación entre universidades, por medio del Consejo de Educación Superior, que aglutina universidades nacionales como privadas, para regular los procesos académicos y la acreditación de títulos, en este consejo se toman decisiones conjuntas en relación al nivel de educación superior. Por otro lado, existe actualmente también una asociación de universidades privadas que ven por su propio desarrollo.

Para lograr una mejor percepción como universidades ellos proponen que se debería: i.- Unificar 
criterios de investigación, ii.- Mejorar la calidad de la investigación que se realiza, iii.- Incentivar la realización de investigaciones conjuntas entre universidades, centros de investigación y profesores y alumnos, iv.- Mejorar el acceso a la información entre universidades.

\section{Grupo Focal de Duelo No 3: 3: Demandante Intermedio y 4: Demandante Final}

De las preguntas y respuestas que dieron las instituciones pertenecientes a los demandantes intermedios y demandantes finales (Díaz, 2012c), lo mas importante es lo que a continuación se describe:

\section{Pregunta general} ¿Cómo percibe la articulación entre ES y el demandante intermedio?

No se percibe vinculación entre las universidades y el mercado laboral por las siguientes razones. i.- El mercado laboral -las empresas- no dan a conocer sus necesidades y que tipo de personal es requerido para cumplir estas exigencias, ii.- Las universidades privadas enfocan su oferta de profesionales para satisfacer las necesidades de empresariales, iii.- Existe más preocupación desde la educación superior por los profesionales que está ofreciendo, que el mismo mercado laboral.

Las universidades no están dando las competencias que el mercado laboral exige, para lo cual sería optimo sacar un perfil en el cual se consideren todas las necesidades que el mercado necesita en el marco del desarrollo del país. Por otro lado, se ve una deficiencia en el uso de tecnologías y el manejo de idiomas, que son otras competencias que deberían tener los profesionales para competir en un mercado laboral más exigente.

\section{3: Demandante Intermedio $\rightarrow$ 4: Demandante Final}

\section{¿Está teniendo un impacto positivo los resultados de ES? ¿Se observa el valor agregado que inducen las IES?}

Existe un problema sobre el empleo, en relación a cuatro aspectos que existen en el país: i.- 
Sub-empleo, ii.- Empleos que no generan mayor productividad, ii.- Sobreoferta de profesionales universitarios en ciertas áreas, iv.- Profesionales en ciertas áreas del desarrollo que son requeridas con urgencia, por ser escasas o porque no se tiene la oportunidad de estudiarlas en el país.

Ya el Plan de Nación plantea lo del empleo como una de los pilares fundamentales del desarrollo del país, sin embargo, falta establecer en cuáles áreas de la producción debe desarrollarse más y que profesionales o áreas de conocimiento son requeridas. Falta aún crear los mecanismos políticos y sectoriales para no solo generar empleo para los profesionales, sino para ubicarlos correctamente en el mercado laboral.

Por otro lado, también las universidades deberían identificar las áreas donde se necesitan un determinado profesional y en qué áreas está saturado, para que la oferta de profesionales vaya de acuerdo a lo que el mercado laboral necesita y poder dar cuotas de oportunidades laborales para todos los profesionales universitarios.

Otro problema adicional a los planteados que también dificulta la buena colocación de profesionales en el mercado laboral, esto es: i.- Profesionales sin graduarse, ocupan puestos de trabajo que deberían ser para profesionales graduados, ii.- Incumplimiento de colegiarse en los colegios profesionales, como requisito para trabajar iii.- Falta de incorporación del título cuando se realizan estudios en el extranjero.

\section{4: Demandante Final $\rightarrow$ 3: Demandante Intermedio}

\section{¿Cómo primeros demandantes de Educación Superior, ¿Qué actividades fundamentales esperan que mejore la universidad?}

Las universidades deberían apoyar la labor docente por medio de estímulos que aseguren su competencia como docentes y criterios mas actualizados de la académica, por medio de: i.- Capacitación continua, ii.- Pasantías, iii.- Becas, iv.- Intercambios con otras universidades, v.- Investigaciones conjuntas, lo que permitiría mejores docentes universitarios, más capaces y desarrollando planes curriculares más exitosos. 
De esta manera, se mejoraría también la capacidad de análisis y la articulación la teoría- práctica y el uso repetitivo de materiales de texto que no se actualizan. Asimismo, los estudiantes universitarios no tienen las actitudes académicas que se requieren, ya sea porque no tienen el nivel o la preparación adecuada, en ese sentido, se debería estimularlos a un mejor desempeño como estudiantes.

Las universidades también tienen su corresponsabilidad en la calidad de la educación superior, siendo las universidades privadas quienes tienen mejor mercadeo para darse a conocer y atraer estudiantes, pero a veces caen en algunos errores que van en detrimento de la educación superior, esto es, que no tienen un efectivo plan curricular, algunas ofrecen dos títulos en poco tiempo, no tienen efectivos programas de docencia.

\section{3: Demandante Intermedio $\rightarrow$ 4: Demandante Final}

\section{¿Quién debería manejar la sobreoferta, la oferta balanceada y la escasa oferta de profesionales?}

Se debería mejorar en las universidades el emprendedurismo y preparar profesionales no solo que sean empleados, sino para que tengan las capacidades necesarias que se necesitan para generar sus propias empresas.

Se debería estimular a los estudiantes y profesionales para tomar capacitaciones extra-curriculares en actividades en las cuales deban prepararse o tengan debilidades, de manera de compensar o aminorar los errores que tienen como producto del sistema educativo hondureño. Se necesita formar profesionales más técnicos a través de un modelo de generación de capacidades y destrezas profesionales.

\section{4: Demandante Final $\rightarrow$ 3: Demandante Intermedio}

\section{¿Existe un margen entre el valor percibido y el costo de formación?}

Existe una descoordinación entre las universidades, por un lado, la UNAH privilegia lo académico y el conocimiento, y por otro lado, las universidades privadas dan más énfasis a lo técni- 
co-empresarial, en este panorama, hay una idea generalizada de que los profesionales de la UNAH se desempeñan mejor que los profesionales del resto de las universidades.

Para el caso de la UNAH, existe una sobresaturación de alumnos matriculados en carreras del área social, y muy poco en las áreas técnicas o de la ciencias básicas. Unido a esta situación, también hay que considerar que se ha descuidado la metodología de enseñanza y la pedagogía con que se imparten las clases.

Se debería establecer convenios y alianzas estratégicas con el sector público y privado para trabajar coordinadamente en actividades de impacto para la sociedad, un ejemplo de esto sería la investigación, de manera que, su ejecución sea conjunta y los resultados sean divulgados ya sea por medio de publicaciones o por medio de conferencias o simposios.

\section{Grupo Focal Integrador de todos los eslabones de la cadena de valor de Edu- cación Superior}

\section{1: Proveedores de Insumos, 2: Educación Superior (Ofertantes),} 3: Demandante intermedio, 4: Demandante Final

Conciliando respuestas y preguntas integrando de todos los eslabones de la cadena de valor de la educación superior, esto es todas las instituciones de la misma (Díaz, 2012d) se concluye lo siguiente:

\section{¿Qué transformaciones considerarían para corregir las deficiencias en educa- ción media como insumo para la educación superior? Todos los eslabones de la cadena de valor de educación superior llegaron a las siguientes recomenda- ciones:}

-Reasignar el presupuesto asignado a la educación, que los recursos financieros para el nivel prebásico y básico sean mayores y para la educación media menor.

- Mejorar el nivel de supervisión de los programas de educación formal para minimizar sus errores y hacerlos más efectivos.

-Diversificar la metodología de enseñanza y los contenidos temáticos e impulsar el método práctico (aprender haciendo).

-Fomentar en el estudiante la investigación, el análisis y escribir ensayos.

-Disminuir la brecha entre escuelas públicas y privadas. 
-Mejorar el modelo educativo, calificar a los docentes no solo en su disciplina y metodologías de enseñanza, sino formar conciencia para un compromiso con la institución y el país.

-Orientar la cooperación internacional hacia la educación formal y no solamente la educación no formal.

\section{2.- Cómo podrían las universidades adaptar sus programas para captar insumos que hoy en día no están entrando en el sistema de ES (personas y proyectos)?}

-Las universidades deben ser más prácticas y crear carreras más técnicas.

-Los estudiantes pueden tener más vinculación a través de tutorías.

-Articular mejor las universidades entre sí para diversas actividades conjuntas.

-Investigar ¿por qué los jóvenes no llegan a la universidad?

-La Prueba de Aptitud Académica (PAA) debe aplicarse para determinar vocación, conocimiento y aptitudes. Se debería descentralizarse los procesos de aplicación de las pruebas de aptitud.

-Formular un proyecto que plantee mejorar el acceso a la educación superior.

\section{3.- ¿Cómo ofrecer un mejor respaldo al estudiante de las Instituciones de Educación Superior?}

-Fortalecer los programas de orientación y asistencia en la escogencia de carrera universitaria para los estudiantes que se postulan para la universidad, especialmente de la UNAH.

-Crear las condiciones que estimulen a los jóvenes para seguir estudios universitarios.

-Ofrecerles reforzamiento, tutorías, bonos, ayuda para el transporte, beca y sobre todo apoyar a los estudiantes de excelencia académica.

-El segundo idioma debe ser requisito para egresar de la universidad.

-Dar espacios de recreación y facilidades de acceso a laboratorios y bibliotecas.

-Cumplir con la normativa legal para que se cumplan los requisitos exigidos en la creación de instituciones de educación superior.

-Establecer alianzas estratégicas con entes de cooperación para mejorar el acceso a los programas de becas universitarias. 


\section{4.- ¿Cómo mejorar la eficacia y eficiencia de los procesos de educación superior?}

Reformular un verdadero plan de desarrollo de la ES para todas las universidades.

-Hacer énfasis en los procesos de certificación y acreditación de carreras, de manera que se cumplan los estándares internacionales. Certificación curricular.

-Tener un programa de fortalecimiento y nivelación para los que han descontinuados estudios. Entonces, incorporar un programa propedéutico.

\section{5.- ¿Cómo se podrían mejorar las fricciones del mercado laboral?}

-Diversificar la oferta educativa e incorporar más clases optativas y no tenerlas de manera obligatoria. -Reorientar la oferta en función de la regionalización que establece el Plan de Nacional.

\section{6.- ¿Qué especialidades técnicas necesita el mercado laboral, que las universi- dades no ofrecen?}

-Electrónica, actuaria y archivista

\section{7.- ¿Cómo se rompería la brecha entre la oferta académica y la demanda laboral para sostener la creciente llegada de empresas multinacionales y compañías locales que fijan su mirada en el boom tecnológico?}

-Realizar investigaciones a nivel regional para determinar áreas de conocimiento a desarrollar.

-Que las tesis de grado se realicen de acuerdo a la demanda de las empresas y la sociedad, previo establecimiento de convenios institucionales.

-Dar seguimiento a los graduados generando mecanismos de apoyo para su inserción en el mercado laboral.

-Preparar al estudiante desde la educación media, para que estimularlos a que estudien carreras que el mercado laboral demanda.

-Invertir en infraestructura y laboratorios que implica la apertura de ciertas carreras orientadas a la diversificación de la oferta. 


\section{Recomendaciones}

Por medio del proceso de consulta que se le hiciera a todos los eslabones de la cadena de valor y suministro de educación superior en los diferentes grupos focales surgieron algunas recomendaciones que ellos mismos dieron, lo cual es válido desde la perspectiva que cada una de los eslabones desde su propio conocimiento y experiencia.

1. En relación al sistema integrado de valor y suministro de la educación superior debe de estandarizarse, integrarse y sistematizarse viendo cada una de sus fases.

2. Inducción vocacional a los estudiantes para la elección de carrera universitaria.

3. Los programas curriculares si están estructurados pero deben renovarse ya que estos no están diseñados de acuerdo a lo que el desarrollo de un país requiere, debe de someterse a un proceso de revisión para actualizarlos y adaptarlos a las verdaderas necesidades del país.

4. Se debe de realizar revisiones curriculares de manera permanente, hay tres aspectos que inciden en dicha actualización orientados a: i.- Actualizaciones e innovaciones de la curricula, ii.- Aplicación de una metodología de la enseñanza teórica con la práctica, iii.- Elementos de emprendedurismo.

5. Hacer un esfuerzo de modernizarlas y aumentarlas de acuerdo a la demanda de estudiantes

6. Los procesos de certificación de programas de pre-grado y post-grado deberían implementarse procesos más ágiles y rápidos que permitan a los graduados certificar mejor y más rápidamente sus títulos.

7. En cuanto a la investigación deberían dar sus resultados, deberían de publicarse y tener accesibilidad de toda la sociedad en su conjunto.

8. Para lograr una mejor percepción como universidades ellos proponen que se debería: i.- Unificar 
criterios de investigación, ii.- Mejorar la calidad de la investigación que se realiza, iii.- Incentivar la realización de investigaciones conjuntas entre universidades, centros de investigación y profesores y alumnos, iv.- Mejorar el acceso a la información entre universidades.

9. Sacar un perfil en el cual se consideren todas las necesidades que el mercado necesita en el marco del desarrollo del país para desarrollar las competencias que el mercado laboral exige.

10. Establecer en cuáles áreas de la producción debe desarrollarse más y que profesionales o áreas de conocimiento son requeridas.

11. Crear los mecanismos políticos y sectoriales para no solo generar empleo para los profesionales, sino para ubicarlos correctamente en el mercado laboral.

12. Las universidades deberían identificar las áreas donde se necesitan un determinado profesional y en qué áreas está saturado, para que la oferta de profesionales vaya de acuerdo a lo que el mercado laboral necesita y poder dar cuotas de oportunidades laborales para todos los profesionales universitarios.

13. Las universidades deberían apoyar la labor docente por medio de estímulos que aseguren su competencia como docentes y criterios mas actualizados de la académica, por medio de: i.Capacitación continua, ii.- Pasantías, iii.- Becas, iv.- Intercambios con otras universidades, v.Investigaciones conjuntas, lo que permitiría mejores docentes universitarios, más capaces y desarrollando planes curriculares mas exitosos.

14. Se debería mejorar en las universidades el emprendedurismo y preparar profesionales no solo que sean empleados, sino para que tengan las capacidades necesarias que se necesitan para generar sus propias empresas.

15. Se debería estimular a los estudiantes y profesionales para tomar capacitaciones extra-curriculares en actividades en las cuales deban prepararse o tengan debilidades, de manera de compensar o aminorar los errores que tienen como producto del sistema educativo hondureño. 
16. Formar profesionales más técnicos a través de un modelo de generación de capacidades y destrezas profesionales.

17. Se debería establecer convenios y alianzas estratégicas con el sector público y privado para trabajar coordinadamente en actividades de impacto para la sociedad, un ejemplo de esto sería la investigación, de manera que, su ejecución sea conjunta y los resultados sean divulgados ya sea por medio de publicaciones o por medio de conferencias o simposios. 


\section{REFERENCIAS}

Diaz Reyes, M. E., compiladora (2012a). Resumen de respuestas grupo focal de duelo: Proveedor de Insumos y Educación Superior [Manuscrito no publicado].

Diaz Reyes, M. E., compiladora (2012b). Resumen de respuestas grupo focal de duelo: Educación Superior y Demandante Intermedio [Manuscrito no publicado].

Diaz Reyes, M. E., compiladora (2012c). Resumen de respuestas grupo focal de duelo: Demandante Intermedio y Demandante Final [Manuscrito no publicado].

Diaz Reyes, M. E., compiladora (2012d). Resumen de respuestas grupo focal integrador: Todos los eslabones del sistema de valor de Educación Superior [Manuscrito no publicado].

Mungaray, A. (2001). Las educación superior y el mercado de trabajo profesional. Revista Electrónica vol3nol/contenido-mungaray.html

Ruiz, J. (2005). La cadena de valor. España. Departamento de Publicaciones del Instituto de Empresa. Recuperado el 10 de febrero de 2013, de http://openmultimedia.ie.edu/OpenProducts/cdv/cdv/ Cadena $\% 20$ de $\% 20$ valor.pdf

Teichler, U. (1999). Research on the relationships between higher education and the world of work: Past achievements, problems and new challenges. Higher Education, 38,169-90. 